\title{
Espiritualidade e bioética nas questões sociais envolvendo a enfermagem
}

\author{
Spirituality and bioethics on social issues involving nursing \\ Espiritualidad y bioética sobre los problemas sociales relacionados con enfermería
}

landerlei Andrade Souza ${ }^{1 *}$, Norma Lopes de Magalhães Velasco Bastos ${ }^{1}$, Alba Benemérita Alves Vilela1, Edite Lago da Silva Sena1 ${ }^{1}$, Rita Narriman Silva de Oliveira Boery ${ }^{1}$, Roseanne Montargil Rocha².

\section{RESUMO}

Objetivo: Refletir sobre a bioética e a espiritualidade e a relação destes campos filosófico-sociais e disciplinar para a formação do enfermeiro. Métodos: Esta investigação configura um estudo de caráter descritivo, narrativo, realizado por meio de pesquisa bibliográfica, utilizando como descritores os vocábulos: "bioética", "enfermagem", "espiritualidade" e "segregação social". Os critérios de inclusão foram reflexão acerca da ética e da espiritualidade na enfermagem que abrangesse toda temática proposta no Brasil. Os critérios de exclusão foram os espaços geográficos incertos, artigos que tratavam de outros países ou que não abordassem o tema proposto correlacionando bioética e espiritualidade com enfermagem. Resultados: contemporaneamente os cuidados prestados pela a enfermagem deve ser embasada nos princípios bioéticos com cunho espiritual afim de proporcionar uma melhor qualidade na assistência. Considerações finais: A bioética e a espiritualidade vêm trazer para os profissionais da saúde um perfil mais humanista, crítico, reflexivo e essencialmente ético.

Palavras chaves: Bioética, Espiritualidade, Enfermagem, Segregação social.

\begin{abstract}
Objective: Reflect about bioethics and spirituality and the relationship of these philosophical discipline and social fields for the formation of the nurse. Methods: This research sets a descriptive narrative, character study, carried out by means of bibliographical research, using as the vocabulary descriptors: "Bioethics", "nursing", "spirituality" and "social segregation". Inclusion criteria were reflection about ethics and spirituality in nursing covering any subject proposed in Brazil. Exclusion criteria were the geographic uncertain spaces, articles that dealt in other countries or that don't cover the topic proposed by correlating bioethics and spirituality with nursing. Results: At the same time the care provided by nursing must be based on the principles of bioethics through spiritual nature in order to provide a better quality in assistance. Final considerations: Bioethics and spirituality come bring to health professionals a more humanist profile, critical, reflective and essentially ethical.
\end{abstract}

Keywords: Bioethics, Spirituality, Nursing, Social segregation.

\section{RESUMEN}

Objetivo: A reflexión sobre la Bioética y la espiritualidad y la relación de estas disciplinas filosóficas y ámbitos sociales para la formación de la enfermera. Métodos: Esta investigación establece un estudio del carácter narrativo, descriptivo, llevado a cabo por medio de investigación bibliográfica, usando como los descriptores de vocabulario: "Bioética", "enfermería", "espiritualidad" y "segregación social". Criterios de inclusión fueron la reflexión sobre la ética y la espiritualidad en enfermería que cubre que cualquier tema propuesto en Brasil. Criterios de exclusión fueron los espacios inciertos geográficos, artículos que tratados en otros países o que no cubre el tema propuesto por la correlación de Bioética y la espiritualidad con enfermería. Resultados: Mientras que la atención brindada por enfermería debe basarse en los principios de la bioética a través de la

1 Universidade Estadual do Sudoeste da Bahia, Jequié - Bahia. * E-mail: ianderleiandrade@gmail.com

2 Universidade Estadual de Santa Cruz, llhéus - Bahia. 
naturaleza espiritual con el fin de brindar un mejor servicio de calidad. Consideraciones finales: Bioética y espiritualidad vienen traer a profesionales de la salud un perfil más humanista, crítico, reflexivo y esencialmente ético.

Palabras clave: Bioética, Espiritualidad, Enfermería, Segregación social.

\section{INTRODUÇÃO}

O conhecimento das relações entre espiritualidade ética e saúde tem crescido muito nas discussões científicas tanto por parte dos pesquisadores e profissionais da saúde como pela população em geral, por isso a espiritualidade vem sendo considerada como novo referencial de estudo para a bioética. Alguns autores afirmam que assim como ocorre com a ética e a moral, a espiritualidade e religiosidade não são sinônimos e não devem ser encarados como um mesmo objeto de estudo. A espiritualidade pode ser definida como a primeira forma implícita de tratar dimensões profundas da subjetividade sem incluir necessariamente crenças (SOUZA, 2012).

Podemos definir a Bioética como uma ética aplicada. Ela procura auxiliar o homem na resolução de potenciais conflitos morais implicados pelas diversas práticas nos campos das ciências da vida e da saúde. A Bioética também pode ser considerada como a ética da ação humana em relação à vida. Sendo assim, a bioética vem também auxiliar na reflexão a respeito da realidade cotidiana e dos conflitos morais que permeiam muitas práticas de saúde (ZANELLA, 2018).

O termo bioética vem como forma de enfatizar dois componentes importantes o conhecimento científico e os valores humanos. Sob o contexto social responder as diversas e inúmeras questões sobre as transformações em diversas áreas do saber e as relações humanas, viabiliza como consequências a possibilidade de atender a uma melhoria no atendimento da enfermagem conseguindo assim uma melhora na qualidade de vida para o ser humano (CAMPOS, 2013).

Souza (2013) vêm trazendo a espiritualidade como elemento que em sua abordagem elenca pontos que podem ajudar a bioética a se firmar como uma ética que se preocupa no uso correto das novas tecnologias na área das ciências da saúde para as soluções adequadas dos dilemas morais por ela apresentados, tornando-a, portanto, um ramo específico da filosofia moral com características próprias.

Um dos grandes enfoques da bioética é o cuidado. Cuidar significa assistir o ser humano em suas necessidades básicas, sendo elas necessidades físicas, sociais, psicológicas e morais. Este deve ser sempre o caráter universal do cuidado. Entretanto, na prática, o cuidado se apresenta de forma diferente, tanto em sua história como em sua contextualização real, portanto, o cuidado é variável e depende de relações que se estabelecem no processo de assistência, tornando-se uma atividade bastante complexa a partir do momento que sai da teoria e vem para a realidade. O cuidado é importante, mas só ele não basta, as reflexões do cotidiano dos indivíduos na sociedade, as quais reagem com as inúmeras tomadas de decisões, são guiadas pelos valores que se estabelecem em cada ser humano, pelas experiências prévias e, em princípios religiosos, morais e éticos (SOUZA ,2012).

O principialismo, bastante difundido na bioética e que pode contribuir bastante para a enfermagem, é definido como uma corrente particular, que fornece um modelo bioético pujante, especialmente no espaço médico, cujo âmago se baseia em quatro princípios: autonomia, beneficência, não maleficência e justiça. $O$ profissional enfermeiro estuda e tem essa preocupação de aprimorar as relações interpessoais, e sendo assim, os profissionais da enfermagem podem contribuir com a bioética na busca do outro, o olhar o outro como um ser integral, o preocupar-se com as necessidades do outro. Desde o início da história da enfermagem, os parâmetros éticos configuram uma questão primordial para sua prática (SCHLEMPER, J.B., 2018). 
A motivação desse estudo é principalmente reunir alguns conceitos da bioética e da espiritualidade e refletir sobre a relação destes campos filosófico-sociais e disciplinar com a formação do enfermeiro. Além disso, o objetivo do presente estudo é relacionar a bioética e a espiritualidade com o desafio de preparar o enfermeiro para as mais diversas realidades a serem encontradas por ele e transformar essa temática tão importante e, ao mesmo tempo tão necessária como uma das principais discussões no ensino da bioética para a construção da excelência na prática do cuidado, a ciência da enfermagem.

\section{METODOLOGIA}

Esta investigação configura um estudo de caráter descritivo, realizado por meio de pesquisa bibliográfica do tipo narrativa, utilizando como descritores os vocábulos: "bioética", "enfermagem", "espiritualidade" e "segregação social". Para o trabalho com a metodologia pesquisa narrativa alguns termos são destacados por Clandinin e Connelly (2011) como, por exemplo, as pessoas que precisam ser entendidas como indivíduos, onde estão sempre em interação e inseridas em um contexto social. Esse conjunto de termos formam um espaço tridimensional para a investigação narrativa. Buscou-se na literatura consagrada disponível, os elementos para a discussão os termos narrados no campo da bioética e espiritualidade e suas implicações no cuidado de enfermagem no Brasil. Como critérios de inclusão foram utilizadas reflexões acerca da bioética e da espiritualidade na enfermagem que abrangessem toda temática proposta. Os critérios de exclusão foram os espaços geográficos incertos, artigos que tratavam de outros países ou que não abordassem o tema proposto correlacionando bioética e espiritualidade com enfermagem. Os artigos encontrados foram do ano de 2013 até o ano de 2018.

\section{REVISÃO DE LITERATURA}

A sociedade vem buscando soluções para várias demandas. Algumas dessas continuam estagnadas por vários fatores, mas tem avançado, cada vez mais, no quesito direitos humanos, encontrando estratégias para resolver inúmeros conflitos, dentre esses, os intergrupais. No entanto, a discriminação e a segregação social ainda são fenômenos presentes nos dias atuais, pois continuam sendo encarados como "um problema do outro" e, portanto, distante de nós. A espiritualidade, nessa perspectiva, se soma às estratégias de resolução desses problemas (MILLANI, 2015).

Os seres humanos são iguais desde seu nascimento, não lhes podendo ser negado qualquer tratamento ou assistência em função de nenhum tipo de discriminação, seja social, racial ou outro fator, preocupando-se com a equidade na distribuição de bens e recursos considerados comuns, numa tentativa de igualar as oportunidades de acesso a estes bens. Deste modo, descrever uma prática ética envolvendo o trabalho e cuidado da enfermagem está diretamente ligado as tensões e conflitos de valores que se tornam fundamentais para a tomada de decisão de ordem ética que tange aos valores morais e aos conhecimentos em relação à profissão (ARRIEIRA, 2018).

Arrieira (2018) ainda afirma que a bioética e a espiritualidade trazem, em sua bagagem, sentidos complexos e ao mesmo tempo densos que criam espaços concretos dentro do exercício profissional da saúde, principalmente na enfermagem que possui um pilar fundamental que é a arte do cuidar. Assim, a espiritualidade vem ganhando espaço significante no processo saúde-doença, apresentado impactos na saúde física e mental da população, melhorando a qualidade de vida dos indivíduos, gerando bem-estar, prevenindo doenças e agravos da saúde e atuando como mecanismo de enfrentamento nas diversas necessidades de saúde.

A ética, dentro do campo da saúde, consiste num arcabouço de princípios para proteção da vida dos indivíduos além de nortear valores fundamentais para a manutenção da integridade das pessoas objetivando evitar a discriminação e a segregação social que podem ocorrer dentro dos ambientes sociais, interagindo nos processos da saúde/doença. Numa perspectiva geral, essa formulação traz a necessidade de o 
profissional de saúde tomar decisões que estão relacionadas não só às questões de ordem técnica ou científica, mas também relacionar as questões sociais e éticas em seu sólido embasamento ético-moral e científico. Em cada situação particular onde se faz necessário a intervenção do profissional de saúde, há a necessidade de um contexto que vai além das diretrizes profissionais, da ciência e da tecnologia, equilíbrio entre a ética, a espiritualidade e o exercício profissional, o que pode facilitar na reflexão sobre valores e princípios pessoais e as contingências de ordem pessoal, profissional e institucional (CAMPOS, 2013).

Souza (2012), traz que a bioética reconhece e caracteriza o valor de todos os seres vivos e encara os humanos como um dos grandes fios que formam a grande teia da vida, onde todos os fios são importantes e inseparáveis, como também coexistem e são coprodutores uns dos outros. Portanto, ao nos dedicarmos a agir eticamente, estaremos buscando saúde e vida e é essa busca que leva o ser humano a um processo contínuo de crescimento. O cuidar eticamente e espiritualmente do outro é uma atitude que leva à reflexão, principalmente quando fazemos referência ao dia a dia do cuidar de pacientes que se diferem entre si, sendo muitos destes, de certa forma, pessoas estigmatizadas e discriminadas pela sociedade.

Mesmo conhecendo os direitos do paciente e a existência de leis que afirmam que todos devam ser tratados de forma igualitária como citado no código de ética dos profissionais de enfermagem, muitos profissionais da saúde tratam os seres humanos de forma indiferente, provavelmente em consequência de uma sociedade conservadora e autoritária, que deixa à margem determinados grupos sociais por considerar esses como tarefa difícil para que haja uma mudança. A atitude ética de qualquer profissional de saúde para com o paciente pode ser facilmente percebida cada vez que ele reconhece seus clientes como pessoas iguais a ele, pessoas que precisam ser ouvidas e compreendidas para que exista uma interação e, por conseguinte, proporcionar um cuidado efetivo (SCHLEMPER, 2018).

O profissional que está cuidando do outro deve, independente de raça, condição sexual, religião, condições econômicas, etnia e estilos de vida, respeitar a pessoa na sua integralidade, com suas opiniões e sua historicidade. Com isso, assumirá uma atitude ética, de respeito e acolhimento do outro. O discernimento entre o profissional e o pessoal, a reflexão em relação as atitudes, crenças e valores fundados no agir livre, consciente e responsável, têm a ver com atitudes adequadas a um verdadeiro profissional comprometido com o seu trabalho e com sua escolha profissional (SOUZA, 2013).

Os dilemas éticos mais vivenciados pelos profissionais de enfermagem são a discriminação, a marginalização e a segregação social em relação aos pacientes. Tendo em vista que o cuidar de pessoas diferentes passa a fazer parte do dia a dia de muitos profissionais da área da saúde, é preciso resgatar a verdadeira essência desse cuidado, para possibilitar maior imparcialidade entre os seres humanos e, com isso, desvelar um mundo com mais valor, presente nos conceitos éticos necessários para se viver em comunidade (CAMPOS, 2013).

Frente aos dilemas éticos vivenciados pelos profissionais de saúde, existe a necessidade de um posicionamento por vezes espiritual, não religioso, de uma qualificação e capacitação atuantes nesse meio. É preciso que os profissionais da enfermagem tenham plena convicção e clareza de sua identidade, colocando sua competência a serviço das necessidades do cuidado a saúde e a vida. Ao cuidar de um paciente, é preciso que o profissional o veja como um ser humano, com suas necessidades básicas, que nesse momento estão afetadas, fragilizadas. Por isso o respeito e a atenção devem estar em primeiro lugar em um plano assistencial (ROCHA, 2017).

A bioética vem mostrar aos profissionais da saúde, um novo perfil, um profissional que deva ser mais humanista, crítico, reflexivo e essencialmente ético, tanto para diminuir problemas como imperícia, negligência e imprudência, quanto para melhorar a qualidade da assistência, através de um cuidado diferenciado, onde pode utilizar, desde conceitos éticos, até a espiritualidade como recurso para alcançar a promoção da saúde, bem como a solução de determinantes sociais que afetam a população (MILLANI, 2015). 
Muitos pacientes que chegam até os profissionais de saúde passam por situações de "despersonalização", que os levam a se sentirem desvalorizados e discriminados, fatos que infringem os preceitos éticos que norteiam a prática do cuidado. Tendo em vista o cuidar como um valor profissional e pessoal, é extremamente importante reconhecer que a bioética não se limita a ética da saúde, mas também a inúmeras outras áreas como a espiritualidade, respondendo questões e problemas que são únicos.

Segundo Evangelista (2016), os enfermeiros entendem que a espiritualidade é muito importante na assistência à saúde, sendo fonte de força, conforto e fé, melhorando o quadro clínico dos pacientes. Na visão dos enfermeiros o apoio familiar, o perdão, o amor, a crença, a fé e a esperança são as principais necessidades espirituais dos pacientes e utilizam a comunicação além de outras práticas como a escuta, a música, a formação de vínculo para entender melhor e atender a estes pacientes.

Gerone (2016) não encontra contradições quando, na doença, o paciente recorre à sua religiosidade/espiritualidade. No entanto, para Alves (2011), médicos, enfermeiros, psicólogos e outros profissionais, apesar de acreditar ser importante integrar a religiosidade/espiritualidade no cuidado hospitalar devido à sua influência no processo saúde/doença, entende haver restrições de alguns profissionais em relação à religiosidade/espiritualidade quando atrapalham o cuidado hospitalar, como crenças e mitos, populares ou dogmáticos. Segundo o autor, esta postura incerta dos profissionais está relacionada com a falta de treinamento e de preparo para lidar com a o contexto religioso/espiritual no contexto do cuidado.

\section{CONSIDERAÇÕES FINAIS}

Oportunamente, observa-se a necessidade de capacitação da equipe de enfermagem quanto a sensibilização e busca de novos valores que direcionem para a reflexão e qualificação do cuidado. Nessa linha, sabendo que a espiritualidade apresenta resposta benéfica no enfrentamento dos problemas e agravos à saúde, ela precisa ser exercida pelos profissionais de saúde nesse cuidado aos usuários. Assim, existem duas funções fundamentais no processo do cuidar, a função técnica que dispõe dos diferentes saberes pessoais e profissionais, que domina um quadro de competências, permitindo recomendar as intervenções necessárias, aumentando as possibilidades do cuidar e, a função ética e humana que esclarece ao usuário acerca do processo do cuidar dos riscos, direitos e oportunidades do tratamento, e, as dificuldades associadas a cada opção.

Com a realização desta revisão bibliográfica, foi possível trazer em outras palavras qual a definição do cuidar, ou seja, caminhar com a pessoa que está sendo cuidada e promover além da saúde um respeito ético e espiritual estabelecendo dessa forma uma função dupla tanto para os profissionais quanto para os usuários. Por fim, destaca-se a necessidade de se produzir conhecimento sobre a temática e a importância da bioética e da espiritualidade para formação dos futuros profissionais de enfermagem e outros profissionais da saúde, o que transforma esses profissionais em sujeitos reflexivos e conscientes, embasados em princípios éticos e morais no exercício de sua profissão.

\section{REFERÊNCIAS}

1. ALVES, MA espiritualidade e os profissionais da saúde em cuidados paliativos. Dissertação de Mestrado em Cuidados Paliativos. Lisboa: Faculdade de Medicina de Lisboa, 2011

2. ARRIEIRA ICO, THOFERHN MB, SCHAEFER OM et al O sentido do cuidado espiritual na integralidade da atenção em cuidados paliativos. Rev. Gaúcha Enferm. [Internet]. 2017 [cited 2018 May 26]; 38(3): e58737. Available from: http://www.scielo.br/scielo.php?script=sci_arttext\&pid=S198314472017000300415\&lng=en .

Epub Apr 12, 2018. http://dx.doi.org/10.1590/1983-1447.2017.03.58737.

3. ARRIEIRA ICO, THOFEHRN MB, PORTO AR, et al. Espiritualidade nos cuidados paliativos: experiência vivida de uma equipe interdisciplinar. Rev. esc. enferm. USP [Internet]. 2018 [cited 2018 May 26]; 52: e 03312. Available from: http://www.scielo.br/scielo.php?script=sci_arttext\&pid=S008062342018000100401\&lng=en. 2018. http://dx.doi.org/10.1590/s1980-220x2017007403312.

Epub Apr 12, 
4. BIONDO CS, FERRAZ MOA, SILVA MLM et al. Espiritualidade nos serviços de urgência e emergência. Rev.

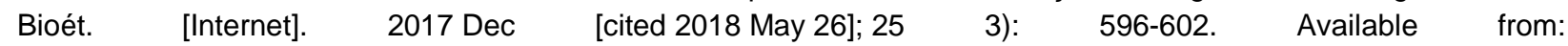
http://www.scielo.br/scielo.php?script=sci_arttext\&pid=S198380422017000300596\&lng=en. http://dx.doi.org/10.1590 /1983-80422017253216.

5. CAMPOS PZ, EL, CABRAL SM. Valores da enfermagem como prática social: uma metassíntese qualitativa. Revista $\begin{array}{llll}\text { Latino-Americana de } \quad \text { Enfermagem } & \text { [Internet]. }\end{array}$ http://www.redalyc.org/articulo.oa?id=281427949007.

6. CLANDININ, D. Jean. CONELLY, F. Michael. Pesquisa narrativa: experiências e história na pesquisa qualitativa. Tradução: Grupo de Pesquisa Narrativa e Educação de Professores ILEEL/UFU. Uberlândia: EDUFU, 2011.

7. EVANGELISTA CB, LOPES MEL, COSTA SFG et al. Espiritualidade no cuidar de pacientes em cuidados paliativos: Um estudo com enfermeiros. Escola Anna Nery 20(1) Jan-Mar 2016. http://www.scielo.br/pdf/ean/v20n1/1414-8145ean-20-01-0176.pdf

8. GERONE, LGT. A religiosidade/espiritualidade na prática do cuidado entre profissionais de saúde. INTERAÇÕES CULTURA E COMUNIDADE, BELO HORIZONTE, BRASIL, V.11 N.20, P. 129-151, JUL./DEZ. 2016. http://periodicos.pucminas.br/index.php/interacoes/article/viewFile/P.1983-2478.2016v11n20p129/10898

9. HEGEL, FW. Enciclopédia das ciências filosóficas. Trad. Paulo Meneses. São Paulo: Loyola, 1995. V. 1.

10. LONGUINIERE ACFDE LA, YARID SD, SILVA ECS. Influência da religiosidade/espiritualidade do profissional de saúde no cuidado ao paciente crítico. Rev Cuid [Internet]. 2018 Apr [cited 2018 May 26]; 9(1): 1961-1972. Available from:

http://www.scielo.org.co/scielo.php?script=sci_arttext\&pid=S221609732018000101961\&lng=en. http://dx.doi.org/10. 15649/cuidarte.v9i1.413.

11. MILLANI H, OLIVEIRA SL. Ética e psicanálise na formação do profissional enfermeiro. Tese (doutorado) Universidade Metodista de Piracicaba, Educação, 2015. Disponible en: http://www.memoria.fahce.unlp.edu.ar/trab_eventos/ev.4487/ev.4487.pdf

12. ROCHA, DD, NASCIMENTO, EC, RAIMUNDO, LP et al. Sentimentos vivenciados pelos profissionais de Enfermagem diante de morte em unidade de terapia intensiva neonatal. Mental - v. 11 - n. 21 - Barbacena-MG - Jul-Dez 2017 - p. 546-560. http://pepsic.bvsalud.org/pdf/mental/v11n21/v11n21a15.pdf

13. SÁ, AC, PEREIRA, LL. Espiritualidade na enfermagem brasileira: retrospectiva histórica. O mundo da saúde São Paulo: 2007: abr/jun 31(2):225-237. Acesso: http://www.saocamilosp.br/pdf/mundo_saude/53/10_Espiritual_enfermagem.pdf

14. SCHLEMPER JBR. Bioética no acolhimento a dependentes de drogas psicoativas em comunidades terapêuticas. Rev. Bioét. [Internet]. $2018 \mathrm{Jan} \quad$ [cited 2018 May 26]; 26(1): 47-57. Available from: http://www.scielo.br/scielo.php?script=sci_arttext\&pid=S198380422018000100047\&lng=en. http://dx.doi.org/10.1590 /1983-80422018261225.

15. SILVA, JB, SILVA LB. Relação entre religião, espiritualidade e sentido da vida. Revista da associação brasileira de logoterapia e análise existencial 3 (2), 203-215, 2014

16. SOUZA VCT, PESSINI L, HOSSNE WS. Bioética, religião, espiritualidade e a arte do cuidar na relação médicopaciente. Revista Bioethikos. 2012;6(2):181-90.

17. SOUZA CFB. Espiritualidade e Bioética. Rev. Pistis Prax., Teol. Pastor., Curitiba, v. 5, n. 1, p. 123-145, jan./jun. 2013. Acesso: http://www.redalyc.org/pdf/4497/449749233006.pdf

18. VASCONCELOS, KM. Convivendo com a alteridade: representações sociais sobre o aluno com deficiência no contexto da educação inclusiva. Recife: O autor, 2008.

19. ZANELLA DC. Humanidades e ciência: uma leitura a partir da Bioética de Van Rensselaer (V. R.) Potter. Interface (Botucatu) [Internet]. $2018 \mathrm{Apr} \quad$ [cited 2018 May 26]; 22(65): 473-480. Available from: http://www.scielo.br/scielo.php?script=sci_arttext\&pid=S141432832018000200473\&lng=en. http://dx.doi.org/10.1590/ 1807-57622016.0914. 